\title{
EFFECT OF in vitro DIGESTION GASTROINTESTINAL OF THE EXTRACT AQUEOU OF LEAVES OF Ugni molinae, ON THE VIABILITY OF COLORECTAL CANCER CELLS
}

\author{
MARCIA AVELLO ${ }^{a}$, EILLEN TORRES $^{a^{*}}$, ROMINA I. CARVAJAL ${ }^{a}$ AND EDGAR PASTENE ${ }^{b}$ \\ ${ }^{a}$ Laboratory of Pharmacognosy, Department of Pharmacy, Faculty of Pharmacy, University of Concepción. Barrio Universitario S/N, PO Box 237, Concepción, \\ Bío Bío, Chile. \\ ${ }^{b}$ Laboratory of Synthesis and Natural Products, Department of Basic Sciences, Faculty of Sciences, University of Bío-Bío. Andrés Bello 720, Chillán, Ñuble, Chile.
}

\begin{abstract}
Aim: This work is aimed to the study the effect of aqueous extracts obtained from leaves of Ugni molinae and their respective products of in vitro gastrointestinal digestion on the viability of colon cancer cells.

Methods: Ugni molinae leaves were used to prepare a 1\%, aqueous extract and with this, an in vitro gastrointestinal digestion was performed, obtaining, fraction products of digestion gastrointestinal (Dgi) and fraction residue. this fraction was identified through liquid chromatography (HPLC-UV) and liquid chromatography-mass spectrometry (HPLC-MS), and then these samples were evaluated in different viability tests, such as trypan blue exclusion, metabolic activity 3-(4,5-dimethylthiazol-2-yl)-2,5-diphenyltetrazolium bromide (MTT) and cytotoxicity by lactate dehydrogenase (LDH), on colorectal cancer cells (CaCo-2) and human embryonic kidney 293 cells (HEK) for a period of 24 and 48 hours.

Results and discussion: The aqueous extracts of leaves of Ugni molinae and their digestion fractions caused inhibition of the viability of Caco-2 cells. In most cases, the effect was concentration-dependent and in particular, the only compound that generated inhibition of the viability in all trials corresponded to the Dgi fraction.

Conclusion: The results are favorable, since it is finally is this product that would act in the gastrointestinal tract, interacting with the target cells. These results are important since in most cases, these compounds had effects on cancer cells Caco-2 and not on HEK cells at the same concentrations used in each trial.
\end{abstract}

Keywords: Digestion gastrointestinal, Viability, Caco-2, Ugni molinae.

\section{INTRODUCTION}

Cancer has become one of the leading causes of death worldwide. Colorectal cancer is the third most commonly diagnosed in men (10\% of total) and the second in women $(9.4 \%$ of total). Still, it maintains a high level of incidence and mortality worldwide and in Chile is the only cancer that remains with a sustained increase [1]. The use of chemotherapy to fight this disease leads to the appearance of side effects [2]. The most frequent side effects of chemotherapy that were reported included fatigue, mouth dryness, nausea, constipation, anorexia, vomiting, pain, sadness and anxiety [3,4]. Side effects of chemotherapy were the main reason for investigations of possible antiproliferative of different natural sources.

In this regard, natural products such as medicinal plants are considered a source of active compounds that can act as prophylactic and complementary treatment in cancer processes if plants are considered in their natural state. On the other hand, the plants are the source of search of anticancer molecules for synthesis or semi-synthesis, against new molecular targets, trying to optimize the conventional treatment by decreasing the doses of compounds with significant adverse effects, to enhance the total anticancer effect and to replace treatments detected as refractory. In this regard, Phenolic compounds, hydrolyzable tannins (gallotannins and ellagitannins) and galloyl flavonoids. mechanisms that dictate in the evolution of cancer studied for phenolic compounds, the regulation of cell proliferation and apoptosis can be mentioned [4,5]. Antiproliferative, cell cycle control (G1 phase), reduction of the cell's migratory potential due to actin cytoskeleton disorganization, and pro-oxidants effects, selective on esophagus and colon cancer, of polyphenols have been reported such as mono-substances and raw extracts. However, few studies have considered neither the effect of the in vitro gastrointestinal digestion nor interactions with other compounds in a complex mixture [6]. These effects would be more important in matrices such the extracts [7]. Therefore, the synergy among these phenolic compounds acting together in a complex mixture with other actives and their metabolites should be assessed as potential therapeutic and anticancer agents.

Given this background, it is reasonable to assume that extracts with important presence of this type of compounds could promote anticancer activity. It is also desirable that the viability of normal cells is not significantly disturbed by these complex mixtures. too have a practical therapeutic importance. These are water soluble compounds present in a large amount of medicinal plants and easily extractable in water, as in the preparation of infusions and decoction preparations.

In this sense Ugni molinae (U. molinae) Turcz. Myrtaceae (Murtilla) is an evergreen plant whose fruits are consumed fresh because of their organoleptic characteristics. U. molinae leaves have been used as infusions by aboriginal people as astringent in case of diarrheas and dysenteries as infusions (1\%). These effects could be related to the chemical composition [8, 9]. In 2013 and as a result of the doctoral thesis [10], new phytochemical findings were reported. The leaves of the population of $U$. molinae from the Biobío region are characterized by having, besides the already reported compounds, phenolic compounds such as gallic derivatives of flavonols, hydrolysable tannins, among others. By being water soluble, these compounds have special importance because they predominate in the popular form of ingestion of leaves of $U$. molinae (infusions and decoctions).

There are no reports on the antiproliferative potential of the leaves of this species, neither the effect of the in vitro gastrointestinal digestion, nor interactions with other compounds in a complex mixture [6] thus, in this study will assess the effect of aqueous extract obtained from leaves of $U$. molinae and their respective products of in vitro gastrointestinal digestion on the viability of human colon cancer cells.

\section{METHODS}

\subsection{Vegetal material and extract obtaining}

U. molinae leaves was collected in the blooming season, in valleys from Biobío Region. Biological material was dehydrated in the shade, at room temperature. In order to obtain the aqueous extracts $(1 \%)$, grinded samples will be processed. These homogenized in a container with water at $80^{\circ} \mathrm{C}$, using a magnetic stirrer for half an hour. Then, samples were filtered through cotton gauze.

\subsection{Products from in vitro gastrointestinal digestion}

Extract aqueous was submitted to an in vitro gastrointestinal digestion, according to Perales et al [10], with a pepsin- $\mathrm{HCl}$ mixture ( $\mathrm{pH} 2.0)$ for two hours, followed by a digestion with pancreatine and bile salts $(\mathrm{pH}$ 6.5). After centrifugation of the digest $\left(3890 \mathrm{~g}, 60 \mathrm{~min}, 4^{\circ} \mathrm{C}\right)$ and to ensure the inactivation of the enzymes as well as the stability of the compounds resulting from the process, the supernatant was acidified with formic acid $(\mathrm{pH} 2.0)$ and filtered by $0.45 \mu \mathrm{m}$ membranes. The residue was treated as described by Saura-Calixto et al [4].

\subsection{Chromatographic analyses}

In order to visualize chromatographic profiles of polyphenols and saponins in extracts, fractions and gastrointestinal digestion products RP-HPLC-UV will be used as described by Pastene et al [11] and Oleszek \& Bialy [12], respectively. 
Fractions and gastrointestinal digestion products will be subjected to analysis by HPLC-ESI-MS/MS [13]. Quantification of main compounds will be carried out by means of the abovementioned methods or by any of them, according to the complexity of the samples.

\subsection{Caco-2 Cells and HEK293 Cells}

These human colorectal cancer cells grew until reaching a density of $10^{4}$ cell $/ \mathrm{cm} 2$ and adherence for $48 \mathrm{~h}$ in a humidified incubator with $5 \% \mathrm{CO}_{2}$ in the air at $37^{\circ} \mathrm{C}$ as described by Cilla et al [6] Cells were cultured in Dulbecco's modified Eagle's medium (DMEM) supplemented with $4.5 \mathrm{~g} / \mathrm{L}$ of glucose, L-glutamine and sodium pyruvate (Corning, New York, USA), 10\% heat inactivated fetal calf serum (Gibco, Thermo Fisher Scientific, Massachusetts, USA) and 1\%penicillin/streptomycin (Hyclone, Thermo Fisher Scientific, Massachusetts, USA). After reaching $80 \%$ to $90 \%$ of confluence, cells tripsinized and seeded in a 96-well plate and the incubated for $24 \mathrm{~h}$ to apply treatments.

Viability in normal cells is evaluated Cells from the SCBN GES-1 (or HEK293-t) of non-malignant gastric cell line will be cultured according to the procedures described by Buresi et al [14] and Ren et al [15], for culture and treatments it was proceeded in the same way as the caco- 2 cells. As reference, compound $\mathrm{H}_{2} \mathrm{O}_{2}$ was used.

\subsection{Sample preparation}

Extract, residue and gastrointestinal digestion product was prepared in PBS1X and sterilized through a $0.22 \mu \mathrm{m}$ filter prior to its addition into the culture medium. If a compound was not completely dissolved in, DMSO was added to reach a final concentration in Wells of $0.1 \%$. For the effect was studied of the concentration and time of incubated, different concentrations ( 0 to 1000) ug / mL final well were prepared and incubated for $24 \mathrm{~h}$ and $48 \mathrm{~h}$.

\subsection{Viability and morphology}

Cells (treated for $24 \mathrm{~h}$ and $48 \mathrm{~h}$ ) viability was measured using Trypan blue dye exclusion assay, for that after the time treats, cells were subjected to a trypsinization process and counted with a Neubauer chamber. Each experiment was carried out in quadruplicate. Furthermore, cells stained with $1 \mu \mathrm{g} / \mathrm{mL}$ of the fluorescent DNA-binding dye Hoechst 33342 and incubated for $1 \mathrm{~h}$ at room temperature to reveal nuclear condensation/aggregation under fluorescence microscope [16].

\subsection{MTT Activity}

Effect of samples was determined by using 3-(4,5-dimethylthiazol-2-yl)-2,5diphenyltetrazolium bromide (MTT). Human cell lines were seeded in a 96-well plate as described above. At the end of treatment ( $24 \mathrm{~h}$ and $48 \mathrm{~h}$ ) with the samples, $100 \mu 1$ of an MTT solution ( $5 \mathrm{mg} / \mathrm{mL}$ in 1xPBS) added to the wells and incubated for $3 \mathrm{~h}$. Then, $500 \mu \mathrm{L}$ of DMSO added to each well after medium removed completely to dissolve the cellular crystalline deposits. The absorbance was measured of at $540 \mathrm{~nm}[16,17]$. Each experiment was carried out in quadruplicate. Results were transformed to percentage of controls, and the IC50 value was obtained by adjusting the dosage-response curve to a sigmoidal model.

\subsection{LDH Cytotoxicity assay}

CaCo-2 and HEK cells were treated with the samples. Subsequently, cell viability was measured through the quantification of the lactate dehydrogenase (LDH) (Kit, BioVision) released in the culture medium. LDH is a cytosolic enzyme that can be released to the culture medium when there is a loss in the cellular membrane integrity, as reflex of loss in cell viability. Thus, LDH quantification in the culture medium is an indirect way to measure cell viability [18]. The results were normalized expressing the value of LDH released to the culture medium in function of total LDH of the cells. To achieve that, cells that remained in the plate after the quantification of LDH release were lysed with Triton $0.01 \%$ to quantify the total LDH presents in cells [19].

\subsection{Statistical analysis}

All quantitative and cell viability determinations conducted in triplicate. Average values $( \pm S D$ ) were calculated. Statistical tests carried out to analyze the correlation between values. Student's $t$ was applied in order to determine the importance of the association of two values. In the case of multiple comparisons, the analysis of variance (ANOVA) was performed, following the Dunnett-test. Differences considered as significant at $\mathrm{p}<0.05$ (versus control).

\section{RESULTS AND DISCUSSION}

In 2013 and as a result of a doctoral thesis [20], new phytochemical findings were informed on leaves of $U$. molinae. In this study, an extract with successive solvents was carried out and it was found that the leaves of the population of $U$. molinae in the Biobío Region are characterized by having phenolic compounds, such as flavonol gallic derivatives, hydrolizable tannins (gallic and ellagitannin compounds), terpenic compounds derived from pentacyclic triterpenic acid of the oleane and ursane nuclei, and glycosylated forms of saponins, among others.

The results of an earlier study carried out in this research team revealed through HPLC-MS analysis that the $1 \%$ aqueous extract of leaves of $U$. molinae (decoction) revealed the presence of phenolic acid derivatives such as hydrobenzoic, gallic and quinic acids; derivatives from flavonoids such as quercetin, heterosides and galloilated, gallotannin and ellagitannin forms. In this type of ectractions, the presence of saponins was found, as revealed in previous studies. Therefore, it is possible to assume that these substances are presented in the form of gennins and non-heterosides, since they are not in the water-soluble form (study recently sent to the Chile Chemistry Journal).

On the other hand, various authors have recorded significant findings about the biological activities of these phenolic and terpenic substances present in leaves of $U$. molinae [7, 8]. In addition, it is important to consider that there is evidence on the anticancer activity of various compounds that are present in this plant [6].

a) $\mathrm{mAC}$
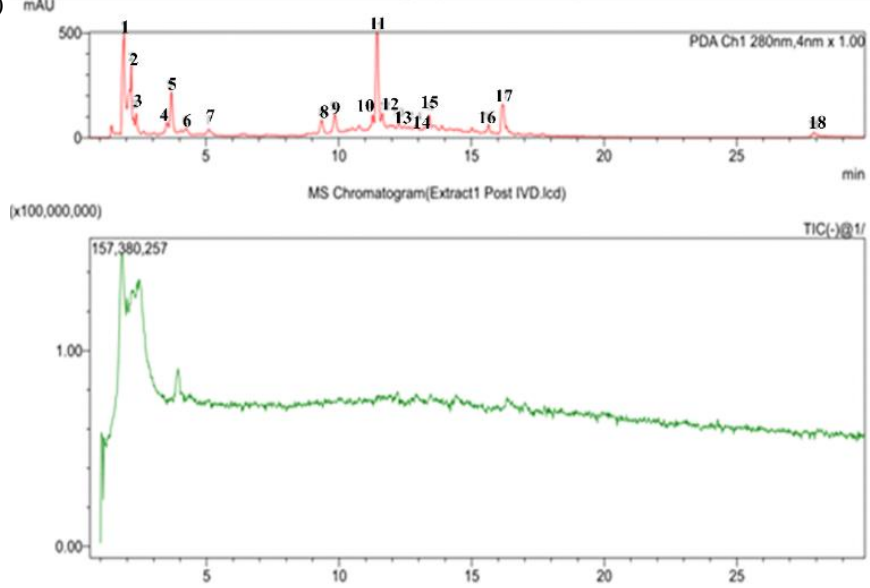

\begin{tabular}{|l|l|l|l|}
\hline Peak & \multicolumn{1}{|c|}{$\mathbf{t}_{\mathbf{R}}(\mathbf{m i n})$} & \multicolumn{1}{|c|}{$\mathbf{~ m / \mathbf { z }}$} & \multicolumn{1}{c|}{ Identification } \\
\hline $\mathbf{1}$ & 1.883 & 341,191 & Caffeic acid hexoside \\
\hline $\mathbf{2}$ & 2.150 & $253,231,191$ & Furostanol derivative \\
\hline $\mathbf{3}$ & 2.367 & $277,231,191$ & Quinic acid \\
\hline $\mathbf{4}$ & 3.533 & $277,231,196$ & Quinic acid \\
\hline $\mathbf{5}$ & 3.700 & $633,277,231$ & HHDP-galloyl hexose \\
\hline $\mathbf{6}$ & 4.233 & 331 & Galloyl glucosa \\
\hline $\mathbf{7}$ & 5.100 & 175,173 & NI \\
\hline $\mathbf{8}$ & 9.350 & $633,451,441,325$ & HHDP-galloyl hexose \\
\hline $\mathbf{9}$ & 9.867 & 449,285 & Kaempferol \\
\hline $\mathbf{1 0}$ & 11.267 & $595,467,405,327,323$, & (Epi)catechin derivative \\
(Procvanidine dimer)
\end{tabular}


b) $\mathrm{MAU}$
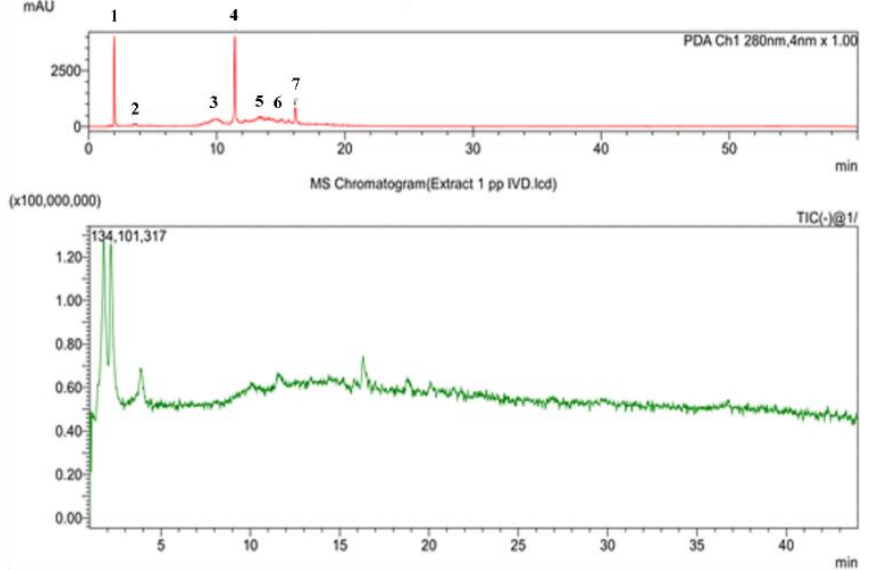

\begin{tabular}{|c|c|l|l|}
\hline Peak & $\mathbf{t}_{\mathbf{R}}(\mathbf{m i n})$ & $\mathbf{m} / \mathbf{z}$ & Identification \\
\hline $\mathbf{1}$ & 1.950 & $383,353,191$ & Quinic acid \\
\hline $\mathbf{2}$ & 3.700 & 633,268 & HHDP-galloyl hexose \\
\hline $\mathbf{3}$ & 9.917 & 783,285 & Pedunculagin I \\
\hline $\mathbf{4}$ & 11.400 & $633,495,469,285$ & HHDP-galloyl hexose \\
\hline $\mathbf{5}$ & 13.500 & $\begin{array}{l}1085,647,542,467, \\
431,421,290\end{array}$ & Cornusiin B / Eucalbanin A \\
\hline $\mathbf{6}$ & 15.583 & $787,539,475$ & Tetragalloyl glucose \\
\hline $\mathbf{7}$ & 16.133 & $651,615,485,449,433$ & Myricetin-O-galloyl deoxyhexoside \\
\hline
\end{tabular}

Figure 1. HPLC-UV chromatogram corresponding to the gastrointestinal digestion products of the $1 \%$ aqueous extract of Ugni molinae and its respective table HPLC-ESI-MS/MS (A). HPLC-UV chromatogram corresponding to the Residue and its respective table HPLC-ESI-MS/MS (B). * Mass spectrometry data in negative polarity. The Electrospray ionization process (ESI). For identification it was contrasted with the fragmentation od phenolic compounds present in libraries prepared from previous identifications and in (http://fiehnlab.ucdavis.edu/staff/kind/Metabolomics/MS-Adduct-Calculator).

For each sample, the UV chromatogram acquired at $280 \mathrm{~nm}$ and the total current of ions acquired in negative polarity are indicated.

The results of this study showed that the $1 \%$ aqueous extract of leaves of $U$. molinae had effect by inhibiting the viability of Caco-q cells through assays of live cell count (trypan blue). Here, a concentration-dependent and statistically significant performance was observed in both treatment times, reaching a viability close to $30 \%$. This implies a $70 \%$ inhibition by the aqueous extract (Figures $2 \mathrm{a}$ and $2 \mathrm{~b}$ ). In the case of HEK cells, there was not a marked effect on changes in cellular viability, except at the highest concentration of $1,000 \mu \mathrm{g} / \mathrm{ml}$ in both treatment times, being obtained a viability of approximately $70 \%$ or an inhibition of 30\%. Therefore, this test demonstrated that this extract has an effect on the inhibition of Caco-2 cells compared to HEK cells. Then, the Hoechst staining microscopy images are consistent with the abovementioned results. In this case, Caco- 2 cells treated for $24 \mathrm{~h}$ with the aqueous extract are affected in their viability since apoptotic cells are observed at the highest concentration used in the essay $(500 \mathrm{ug} / \mathrm{ml})$ compared to untreated cells. In the case of HEK cells, there are no changes respect to control, when they were treated with both concentration of the aqueous extract (Figure 3).

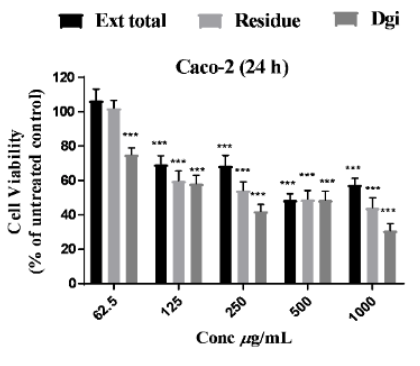

a

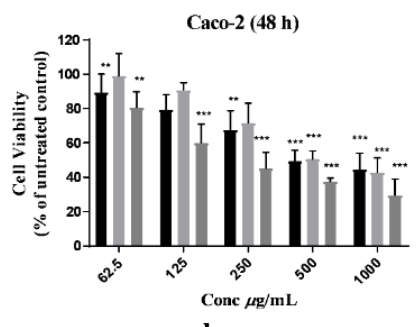

b

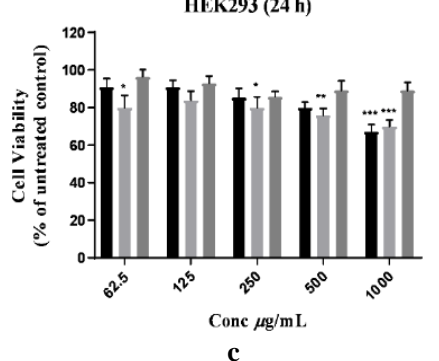

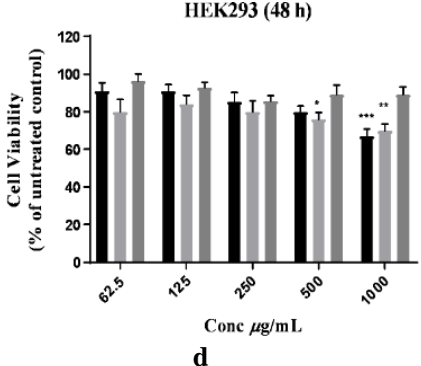

d

Figure 2. Effect of $U$. molinae extract, residue fraction and gastrointestinal digestion on the viability of Caco-2 (A and B) and HEK293 (C and D) cells through trypan blue assay. The cells were treated during 24 and $48 \mathrm{~h}$.

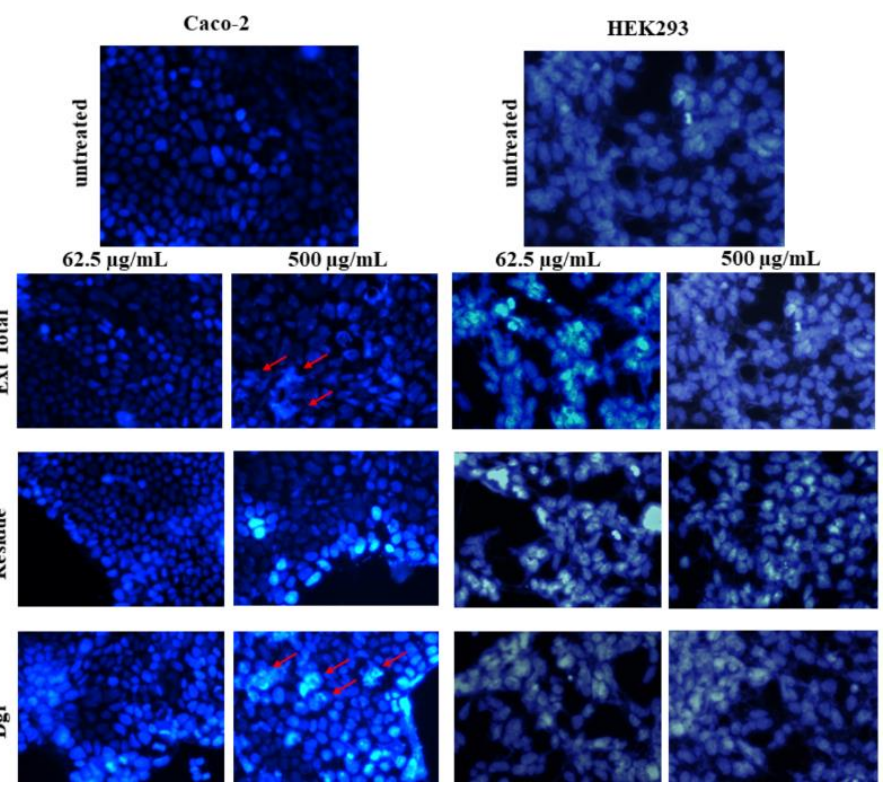

Figure 3. Fluorescent micrography of the extract of $U$. molinae, residue and gastrointestinal digestion product on cellular morphology through the Hoechst staining test in Caco-2 and HEK cells during $24 \mathrm{~h}$ treatment (no scale).

On the other hand, in the essays of MTT enzymatic activity it can be observed that this extract has an effect on the inhibition of the succinate dehydrogenase enzymatic activity of Caco- 2 cells only after $48 \mathrm{~h}$ of treatment, with an effect depending on the concentration. This effect is only observed at higher concentrations, inhibiting more than $50 \%$ of the activity (Figure $4 \mathrm{a}$ and $4 \mathrm{~b}$ ). In addition, an 1C50 of $983 \mu \mathrm{g} / \mathrm{ml}$ was obtained (Table 1). Regarding the lactate dehydrogenase (LDH) enzyme, only a toxic effect close to $20 \%$ in Caco-2 colorectal cancer cells was observed in both treatment times. Therefore, this compound would not be considered toxic in the cells (Figure 5a and 5b). In the case of HEK cells for both enzymatic activity tests, there was a little decrease of the MTT activity after $48 \mathrm{~h}$ of treatment with the extract, reaching an activity of $70 \%$ to $80 \%$, or an inhibition of $20 \%$ to $30 \%$ at the highest concentration used. Although in the LDH test, the cytotoxicity reaches $20 \%$ at $48 \mathrm{~h}$, in these tests it was not possible to determine an 1C50 for the compounds (Figure 4c, 4d, 5c and $5 d)$.

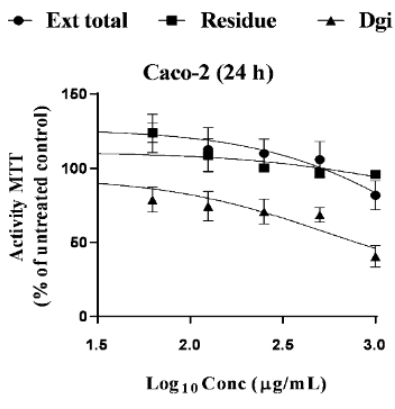

a

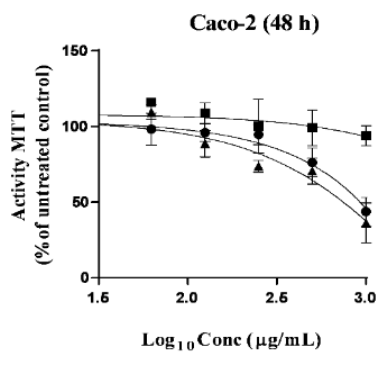

b 

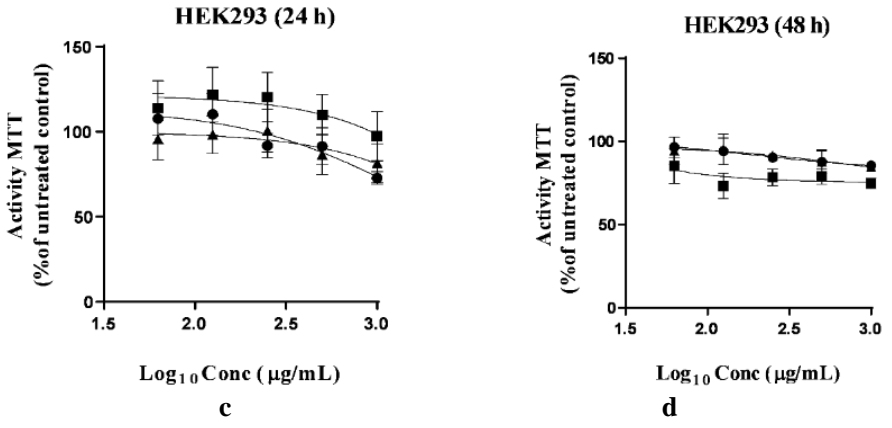

Figure 4: Effect of the total extract of $U$. molinae, residue and gastrointestinal digestion product on the viability of Caco-2 and HEK cells through MTT (A, B, C D). Treatments were carried out during 24 and $48 \mathrm{~h}$.

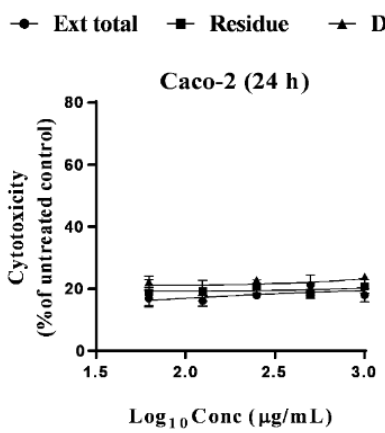

a

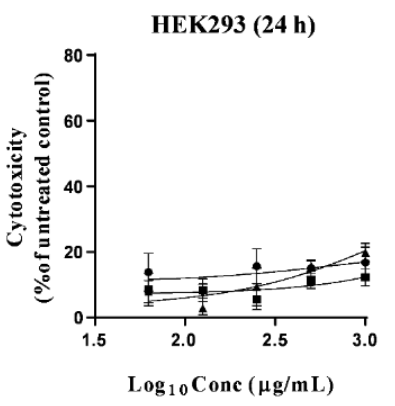

c

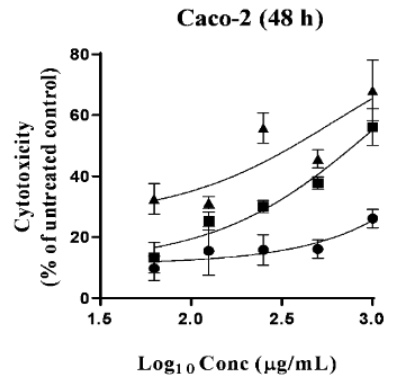

b

HEK293 (48 h)

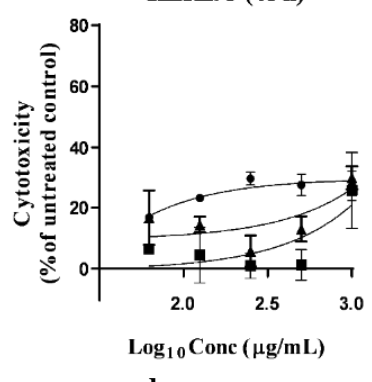

d
Figure 5: Effect of the total extract of $U$. molinae, residue and gastrointestinal digestion product on the viability of Caco-2 and HEK through LDH assays. Treatments were carried out during 24 and $48 \mathrm{~h}$.

Table 1: IC50 values for each assay corresponded to the $95 \%$ confidence limit, which are shown in the table under the curves.

\begin{tabular}{|c|c|c|c|c|c|c|c|c|}
\hline & \multicolumn{8}{|c|}{ IC50 $\mu \mathrm{g} / \mathrm{mL}$} \\
\hline & \multicolumn{4}{|c|}{ MTT } & \multicolumn{4}{|c|}{ LDH } \\
\hline & \multicolumn{2}{|l|}{ Caco-2 } & \multicolumn{2}{|c|}{ HEK293 } & \multicolumn{2}{|c|}{ Caco-2 } & \multicolumn{2}{|c|}{ HEK293 } \\
\hline & $24 \mathrm{~h}$ & $48 \mathrm{~h}$ & $24 \mathrm{~h}$ & $48 \mathrm{~h}$ & $24 \mathrm{~h}$ & $48 \mathrm{~h}$ & $24 \mathrm{~h}$ & $48 \mathrm{~h}$ \\
\hline Ext total & $<1000$ & $983 \pm 10$ & $<1000$ & $<1000$ & $<1000$ & $<1000$ & $<1000$ & $<1000$ \\
\hline Residue & $<1000$ & $<1000$ & $<1000$ & $<1000$ & $<1000$ & $999 \pm 16$ & $<1000$ & $<1000$ \\
\hline Dgi & $598 \pm 8$ & $615 \pm 8$ & $994 \pm 10$ & $<1000$ & $<1000$ & $613 \pm 18$ & $<1000$ & $<1000$ \\
\hline
\end{tabular}

Data are shown as means \pm SD from three independent experiment $(n=3)$.

Although there are no reports about the antiproliferative potential of the leaves of $U$. molinae, there is information about its fruits. Flis et al [21] observed antiproliferative properties (colon cancer cell lines) that are correlated with their antioxidant levels, mainly given by phenolic-type compounds, such as flavonols and tannins. The results are consistent with others that showed that lyophilized berried in the diet inhibit chemically induced cancer of the esophagus in rodents by $30 \%$ to $60 \%$, and colon cancer up to $80 \%$. Other studies indicate that the phenolic compounds, hydrolizable tannins (gallotannins and elagitannins) and galloyl flavonoids present mechanisms that dictate the evolution of cancer. Among them, the following can be mentioned: regulation of cell proliferation and apoptosis [4, 5], antiproliferative effects of the cell cycle (phase G1), reduction of the migratory phase of the cell due to the disorganization of the actin cytoskeleton, and selective prooxidant effects on the esophagus and colon cancer. Based on this evidence, it is possible to assume that the antiproliferative activity of the aqueous extract of $U$. molinae presented in this study is mainly due to the presence of these compounds. In addition, by being soluble in water, these compounds have special importance, because they predominate in the popular form ingestion of leaves of U. molinae (infusions and decoctions). In this sense, a study showed that the extracts obtained at higher temperatures of blackcurrant showed a stronger inhibition of the proliferation of HT-29 cells than extracts obtained at lower temperatures. These findings would indicate that this case that the decoction of leaves of $U$. molinae performed in this study, unlike an infusion, could generate a greater presence of compounds with effect on the inhibition of the viability of cancer cells. This effect might be due to the decomposition of complex polyphenols at higher temperatures, making them more accessible to the cells. Some flavonoids such as quercetin also have a wide range of biological effects that are considered beneficial for health treatment, mainly as anticancer [5].

It is important to note that whereas there is evidence of the anticancer activity of several compounds that are present in this plant, very few studies have considered the effect of in vitro gastrointestinal digestion and the changes that may be generated in the components and their antiproliferative effects.

For this reason, in this study the in vitro gastrointestinal digestion was generated from the $1 \%$ aqueous extract of leaves of $U$. molinae belonging to the soluble fraction. Similarly, the resulting residue at the end of the process corresponded to the insoluble fraction. Initially, tests were carried out to obtain the identification of the compounds present in the fractions generated as digestion products. Figure 1 shows the HPLC-UV and HPLC-MS/MS pictograms of the products generated after the in vitro digestion (gastrointestinal digestion and residue). These are mostly derived from the semisynthetic pathway of chikimic acid and phenolic derivatives.

The products that are generated from a digestion would be the most important in this study, since these fractions, which are products of the metabolism, would act directly in the organism generating an interaction with the white cell, Therefore, they could present different active principles having more potent antiproliferative biological effects than the aqueous extract.

In this study, the products of the gastrointestinal digestion showed for the assays on Caco- 2 cells that the residue had effect by inhibiting the viability of these cells through the live cell count test (trypan blue). Thus, a concentrationdependent performance was observed in both treatment times, reaching a viability close to $50 \%$. The Dgi product had a similar performance, though reaching lower viability values of around $30 \%$. This implies $70 \%$ inhibition at higher concentrations. IN addition, it should be noted that inhibition of viability started to be observed even at lower concentrations compared to the rest of the samples (Figure 2a and 2b). In this case, this last product is the one that generated a greater inhibition pf viability, being observed the most powerful effect in all the analyzed concentrations.

In the case of HEK cells, there was no marked effect on viability, except for the residue fraction, where at the highest concentration of $1000 \mu \mathrm{g} / \mathrm{ml}$, a viability close to $80 \%$ was observed at 24 and $48 \mathrm{~h}$. On the other hand, the Dgi product was unable to generate a marked change in viability, staying close to $100 \%$ (Figure $2 \mathrm{c}$ and $2 \mathrm{~d}$ ). In these tests, mostly Dgi had a marked effect on the death of Caco-2 cells and on the contrary, the same was not observed in HEK cells, where the viability was maintained in all cases. The microscopy images performed by Hoechat staining are consistent with the aforementioned tests, since they show that Caco- 2 cells treated for $24 \mathrm{~h}$ with the residue have negative effect on viability because some apoptotic cells are observed at the highest concentration used in this case, compared to untreated cells. The Dgi fraction also shows negative effect on viability, with several cells being observed in apoptosis compared to the rest of the samples and to the control.

In the case of HEK cells, there are no changes respect to control, when treated with both concentration of the residue fraction and Dgi (Figure 3). 
With respect to tests on enzymatic activity, the residue had no effects on the inhibition of MTT enzymatic activity of Caco-2 cells in any case. In HEK cells at $48 \mathrm{~h}$ treatment, the enzymatic activity was slightly affected, staying between $80 \%$ and $90 \%$. In this case the results are not promising since the residue is unable to generate effects in the increase of the metabolic activity of Caco-2 cells, although mild changes were observed in HEK (Figure 4a, 4b, 4c and 4d). Therefore, this metabolic target would not be the mechanism by which this fraction generates antiproliferative effects in cancer cells. In LDH cytotoxicity tests, in the case of Caco- 2 cells at $24 \mathrm{~h}$ treatment, no marked changes were observed, being maintained the toxicity close to $20 \%$. At $48 \mathrm{~h}$ of treatment, this fraction generated a concentration-dependent cytotoxicity, reaching a value close to $60 \%$ (Figure $5 \mathrm{a}$ and $5 \mathrm{~b}$ ), being capable to maintain only in this case, a calculated IC 50 of $999 \mu \mathrm{g} / \mathrm{ml}$ (Table 1). In HEK cells, cytotoxicity was very low, close to $10 \%$ at 24 and $48 \mathrm{~h}$ (Figure $5 \mathrm{c}$ and $5 \mathrm{~d}$ ).

In the case of the Dgi fraction, in Caco-2 cells there was a greater effect by inhibiting the MTT enzymatic activity, where a concentration-dependent is observed, with values that reached $40 \%$ of activity. This implies an inhibition of this enzymatic activity of $60 \%$, also highlighting further inhibition from lower concentrations (Figure $4 \mathrm{a}$ and $4 \mathrm{~b}$ ). This is for both treatment times, with an IC50 of 598 and $615 \mu \mathrm{g} / \mathrm{ml}$ at 24 and $48 \mathrm{~h}$ (Table 1), being observed a slight decrease and reaching $80 \%$ of activity. In this case, again the results of MTT enzymatic activities are partly consistent with those observed in cell counts and microscopy images by Hoechst staining, since by decreasing the count of living cells, Thus, it is not uncommon to assume that MTT metabolic activity decreases with respect to control without compound.

For cytotoxicity tests through $\mathrm{LDH}$, in Caco- 2 cells at $24 \mathrm{~h}$ there are no marked cytotoxic effect, being remained under $20 \%$. On the other hand, at $48 \mathrm{~h}$ a $70 \%$ cytotoxicity was reached, with a calculated IC50 of $613 \mu \mathrm{g} / \mathrm{ml}$ (Figure 5a and 5b). In the case of HEK cells, cytotoxicity remains under $20 \%$ (Figure $5 c$ and $5 d$ ).

According to reports, the high molecular weight, degree of polymerization and low lipophilicity can be responsible of the limited availability of these compounds in gastrointestinal conditions [1]. Therefore, the most lipophilic compounds are absorbed in the first third of the long intestine, whereas the most hydrophilic would be eliminated though the rectum. Thus, the latter would be the most active compounds that would reach the colon. For example, in the gastrointestinal tract, hydrolizable tannins and galloid flavonols are hydrolyzed to release products by $\mathrm{pH}$. Gallotannins are affected to a greater extent in the large intestine through enzymatic metabolism. These are reduced to gallic derivatives [23, 24].

The formation of different metabolites depends on the modifications that their precursors have previously experienced in the large intestine [24]. Based on this, it should be mentioned that these fractions by being metabolites resulting from gastrointestinal digestion have different mechanisms of action than those of the initial extract. In addition, this mechanism is not unique since it is a complex mixture that contains metabolites that interact in different forms of attack to the target cell.

Therefore, phenolic compounds and their metabolites may develop antiinflammatory, antibiotic, antioxidant and anticancer properties in the gastrointestinal mucosa [25]. This therapeutic importance will depend on the time spent associated with absorption and the biological effects developed in the gastrointestinal tract [2]. In addition, they also have practical therapeutic importance as these are water-soluble compounds present in a large number of medicinal plants and easily removable in water, as in the preparation of infusions and decoction preparations.

This study provides a real knowledge about the gastrointestinal effect of an aqueous extract of a native plant, commonly used as decoction or infusions, on colorectal cancer cells.

Finally, for the results obtained in $\mathrm{CaCo}-2$ cancer cells, it is possible to assume that the $1 \%$ extract at and the fractions resulting from digestion have mechanisms on different cellular targets. In general, in most cases the effect was concentration-dependent and particularly, the only fraction that generated inhibition of viability in all tests performed was the Dgi fraction, which favors these results since it is finally this product that act in the gastrointestinal tract, managing to interact with the target cell.

In conclusion, these results are important since in most cases the samples used had effects on cancer cells and not on HEK cells at the same concentrations used in each trial.

\section{ACKNOWLEDGMENTS}

This work was supported by the National Scientific and Technological Commission, Chile, FONDECYT Program [Grant number 11160275].

\section{REFERENCES}

1. L. Itriago, N. Silva, G. Cortes, Rev. Med. Clin. Condes. 24, 531-552, (2013).

2. M. Polikandrioti, E. Gerasimou, G. Kotronoulas, A. Tsami, E. Evagelou, H. Kyritsi, Nosileftiki. 49, 377-386, (2010).

3. Guía Clínica AUGE, S.d.S.P, Mins. Gob. Chil.1-62, (2014).

4. F. Saura-Calixto, J. Serrano, I. Goñi, Food Chem. 101, 492-501, (2007).

5. N. Seeram, L. Adams, S. Henning, Y. Niu, Y. Zhang, M. Nair, J. Nutr. Biochem. 16, 360-367, (2005).

6. González-Sarrías, A., Azorín-Ortuño, M., Yáñez-Gascón, M.J., Tomás-Barberán, F.A., García-Conesa, M.T., J. Espín, J. Agr. Food Chem. 57, 5623-5632, (2009).

7. M. Suwalsky, P. Orellana, M. Avello, F. Villena, Food Chem. Toxicol. 45, 130-135, (2007).

8. M. Avello, E. Pastene, E. Bustos, M. Bittner, J. Becerra, RBFAR. 23, 44-50, (2013).

9. A. Cilla, A. González-Sarrías, F. Tomás-Barberán, J. Espín, R. Barberá, Food Chem. 114, 813-820, (2009).

10. S. Perales, R. Barberá, M. Lagarda, R. Farré, J. Agric. Food Chem. 53, 3721-3726, (2005).

11. E. Pastene, H. Speisky, M. Troncoso, J. Alarcón, G. Figueroa, J. Agric. Food Chem. 57, 7743-7749, (2009).

12. W. Oleszek, \& Z. Bialy, J. Chromatogr. A. 1112, 78-91, (2006).

13. M. Cho, L. Howard, R. Prior, J. Clark, J. Sci. Food Agric. 84,1771-1782, (2004).

14. M. Buresi, E. Schleihauf, N. Vergnolle, A. Buret, J. Wallace, M. Hollenberg, W. MacNaughton, Am. J. Physiol. Gastrointest. Liver Physiol. 281, G323-332, (2001).

15. J. Ren, H-J. Huang, Y. Gong, S. Ywe, Li-M. Tang, S. Cheng, Cell \& Bioscience. 4, 26, (2014)

16. M. Karagozlu, F. Karadeniz, C. Kong, S. Kim, Carbohydr. Polym. 87, 1383-1389, (2012).

17. M. Bermúdez-Soto, M. Larrosa, J. García-Cantalejo, J. Espín, F. Tomás Barberán, García- M. Conesa, J. Nutr. Biochem. 18, 259-271, (2007).

18. S. Kamo, S. Suzuki, T. Sato, Nutrition. 30, 596-601, (2014).

19. G. Wang, J. Zhang, A. Dewilde, A. Pal, D. Bello, J. Therrien, S. Braunhut, K. Marx, Toxicology. 299, 99-111, (2012).

20. M. Avello, Tesis para optar al grado de Doctor en Ciencias Biológicas, área Botánica, (2013).

21. S. Flis, Z. Jastrzebski, J. Namiesnik, P. Arancibia, F. Toledo, H. Leontowicz, M. Leontowicz, M. Suhaj, S. Trakhtenberg, S. Gorinstein, J. Pharm. Biomed. Anal. 62, 68-7, (2012).

22. M. Dall'Asta, L. Calani, M. Tedeschi, L. Jechiu, Nutrition. 28,197-203, (2012).

23. M. Krook \& A. Hagerman, Food Res. Int. 49,112-116, (2012).

24. O. Andersen, K. Markham, Flavonoids: Chemistry, Biochemistry and Applications. CRC Press, USA, (2006).

25. M. Selma, J. Espín, F. Tomás-Barberán, J. Agr. Food Chem. 57,6485-6501, (2009). 